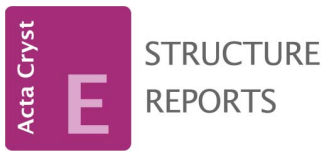

OPEN $\curvearrowright$ ACCESS

ISSN $1600-5368$

\section{Crystal structure of $\left\{2,2^{\prime}\right.$-[ethylenebis- (nitrilomethanylylidene)]diphenolato- $\kappa^{4} \mathrm{O}, \mathrm{N}, \mathrm{N}^{\prime}, \mathrm{O}^{\prime}$ \}oxidovanadium(IV) methanol monosolvate}

\author{
Rachel E. Hsuan, Jemma E. Hughes, Thomas H. Miller, \\ Nabila Shaikh, Phoebe H. M. Cunningham, Alice E. \\ $\mathrm{O}^{\prime}$ Connor, Jeremiah P. Tidey and Alexander J. Blake*
}

School of Chemistry, The University of Nottingham, University Park, Nottingham NG7 2RD, England. *Correspondence e-mail: a.j.blake@nottingham.ac.uk

Received 19 October 2014; accepted 22 October 2014

Edited by J. F. Gallagher, Dublin City University, Ireland

Two independent molecules of the title solvated complex, $\left[\mathrm{V}\left(\mathrm{C}_{16} \mathrm{H}_{14} \mathrm{~N}_{2} \mathrm{O}_{2}\right) \mathrm{O}\right] \cdot \mathrm{CH}_{3} \mathrm{OH}$, also known as $\left[N, N^{\prime}\right.$-bis(salicylidene)ethylenediamine]oxidovanadium(IV) or vanadyl salen, crystallize in the asymmetric unit. Each disordered methanol solvent molecule [occupancy ratios 0.678 (4):0.322 (4) and 0.750 (5):0.250 (5)] is linked to a [N, $N^{\prime}$-bis(salicylidene)ethylenediamine]oxidovanadium(IV) molecule by an $\mathrm{O}-\mathrm{H} \cdots \mathrm{O}$ hydrogen bond and to others by $\mathrm{C}-\mathrm{H} \cdots \mathrm{O}$ hydrogen bonds. The resulting extended structure consists of a bilayer of molecules parallel to the $a b$ plane. Despite the fact that solvates are common in complexes derived from substituted analogues of the $N, N^{\prime}$-bis(salicylidene)ethylenediamine ligand, the title solvate is the first one of $\left[N, N^{\prime}\right.$-bis(salicylidene)ethylenediamine]oxidovanadium(IV) to be structurally characterized. The two vanadyl species have very similar internal geometries, which are best characterized as distorted square-based pyramidal with the vanadium atom displaced from the $\mathrm{N}_{2} \mathrm{O}_{2}$ basal plane by 0.5966 (9) $\AA$ in the direction of the doubly-bonded oxide ligand.

Keywords: crystal structure; oxidovanadium(IV); $2,2^{\prime}$-[ethylenebis(nitrilomethanylylidene)]diphenolate; $N, N^{\prime}$-bis(salicylidene)ethylenediamine; hydrogen bonding.

CCDC reference: 1030592

\section{Related literature}

The literature reports three structure determinations on the unsolvated title complex, also known as [N, $N^{\prime}$-bis(salicylidene)ethylenediamine]oxidovanadium(IV). The first was in the monoclinic space group $P 2_{1} / c$ by Riley et al. (1986), the second by Li et al. (2004) in the triclinic space group $P \overline{1}$ and the third in the monoclinic space group $P 2_{1}$ (Wang et al., 2008). All three determinations were carried out at ambient temperature. According to the Cambridge Structural Database (Groom \& Allen, 2014) no solvates of [ $N, N^{\prime}$-bis(salicylidene)ethylenediamine]oxidovanadium(IV) have been reported previously, although these are common for substituted analogues of the $N, N^{\prime}$-bis(salicylidene)ethylenediamine ligand.

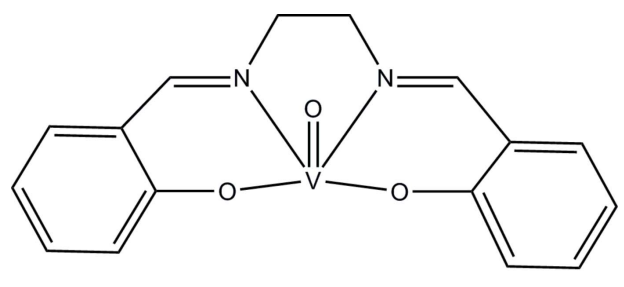

\section{Experimental}

$\mathrm{CH}_{3} \mathrm{OH}$

2.1. Crystal data

$\left[\mathrm{V}\left(\mathrm{C}_{16} \mathrm{H}_{14} \mathrm{~N}_{2} \mathrm{O}_{2}\right) \mathrm{O}\right] \cdot \mathrm{CH}_{4} \mathrm{O}$

$M_{r}=365.27$

Orthorhombic, Pna2 ${ }_{1}$

$a=12.9597$ (4) $\AA$

$b=8.8616(2) \AA$

$c=28.5426(7) \AA$

\subsection{Data collection}

Agilent GV1000 diffractometer with an Atlas CCD detector

Absorption correction: gaussian (CrysAlis PRO; Agilent, 2013)

$T_{\min }=0.480, T_{\max }=0.959$

\subsection{Refinement}

$R\left[F^{2}>2 \sigma\left(F^{2}\right)\right]=0.037$

$w R\left(F^{2}\right)=0.105$

$S=1.04$

4634 reflections

479 parameters

32 restraints

$\mathrm{H}$-atom parameters constrained

Table 1

Selected geometric parameters $\left(\AA,^{\circ}\right)$.

\begin{tabular}{lrlr}
\hline $\mathrm{V} 1-\mathrm{O} 1$ & $1.9155(17)$ & $\mathrm{V} 2-\mathrm{O} 4$ & $1.9164(16)$ \\
$\mathrm{V} 1-\mathrm{O} 2$ & $1.9429(16)$ & $\mathrm{V} 2-\mathrm{O} 5$ & $1.6089(16)$ \\
$\mathrm{V} 1-\mathrm{O} 3$ & $1.6070(17)$ & $\mathrm{V} 2-\mathrm{O} 6$ & $1.9314(16)$ \\
$\mathrm{V} 1-\mathrm{N} 1$ & $2.0597(19)$ & $\mathrm{V} 2-\mathrm{N} 3$ & $2.062(2)$ \\
$\mathrm{V} 1-\mathrm{N} 2$ & $2.051(2)$ & $\mathrm{V} 2-\mathrm{N} 4$ & $2.060(2)$ \\
& & & \\
$\mathrm{O} 2-\mathrm{V} 1-\mathrm{N} 2$ & $87.30(8)$ & $\mathrm{O} 6-\mathrm{V} 2-\mathrm{N} 4$ & $87.20(8)$ \\
$\mathrm{O} 2-\mathrm{V} 1-\mathrm{N} 1$ & $150.78(7)$ & $\mathrm{O} 6-\mathrm{V} 2-\mathrm{N} 3$ & $150.79(7)$ \\
$\mathrm{O} 3-\mathrm{V} 1-\mathrm{O} 2$ & $105.76(8)$ & $\mathrm{O} 5-\mathrm{V} 2-\mathrm{O} 6$ & $105.81(8)$ \\
$\mathrm{O} 3-\mathrm{V} 1-\mathrm{N} 2$ & $107.35(9)$ & $\mathrm{O} 5-\mathrm{V} 2-\mathrm{N} 4$ & $107.66(8)$ \\
$\mathrm{O} 3-\mathrm{V} 1-\mathrm{O} 1$ & $113.39(8)$ & $\mathrm{O} 5-\mathrm{V} 2-\mathrm{O} 4$ & $113.60(8)$ \\
$\mathrm{O} 3-\mathrm{V} 1-\mathrm{N} 1$ & $102.90(8)$ & $\mathrm{O} 5-\mathrm{V} 2-\mathrm{N} 3$ & $102.81(8)$ \\
$\mathrm{N} 2-\mathrm{V} 1-\mathrm{N} 1$ & $78.80(8)$ & $\mathrm{N} 4-\mathrm{V} 2-\mathrm{N} 3$ & $78.63(8)$ \\
$\mathrm{O} 1-\mathrm{V} 1-\mathrm{O} 2$ & $86.60(7)$ & $\mathrm{O} 4-\mathrm{V} 2-\mathrm{O} 6$ & $86.66(7)$ \\
$\mathrm{O} 1-\mathrm{V} 1-\mathrm{N} 2$ & $138.94(8)$ & $\mathrm{O} 4-\mathrm{V} 2-\mathrm{N} 4$ & $138.40(8)$ \\
$\mathrm{O} 1-\mathrm{V} 1-\mathrm{N} 1$ & $87.26(8)$ & $\mathrm{O} 4-\mathrm{V} 2-\mathrm{N} 3$ & $87.25(7)$ \\
\hline
\end{tabular}

$$
\begin{aligned}
& V=3277.92(15) \AA^{3} \\
& Z=8 \\
& \mathrm{Cu} K \alpha \text { radiation } \\
& \begin{array}{l}
\mu=5.27 \mathrm{~mm}^{-1} \\
T=120 \mathrm{~K}
\end{array} \\
& 0.31 \times 0.14 \times 0.05 \mathrm{~mm} \\
& \\
& 7416 \text { measured reflections } \\
& 4634 \text { independent reflections } \\
& 4350 \text { reflections with } I>2 \sigma(I) \\
& R_{\text {int }}=0.025
\end{aligned}
$$

$\Delta \rho_{\max }=0.42 \mathrm{e} \AA^{-3}$

$\Delta \rho_{\min }=-0.29 \mathrm{e}^{-3}$

Absolute structure: Flack (1983). 1395 Friedel pairs

Absolute structure parameter: 0.066 (9) 
Table 2

Hydrogen-bond geometry $\left(\AA,^{\circ}\right)$.

\begin{tabular}{|c|c|c|c|c|}
\hline$D-\mathrm{H} \cdots A$ & $D-\mathrm{H}$ & $\mathrm{H} \cdots A$ & $D \cdots A$ & $D-\mathrm{H} \cdots A$ \\
\hline $\mathrm{C} 14-\mathrm{H} 14 \cdots \mathrm{O}^{\mathrm{i}}$ & 0.93 & 2.48 & $3.368(4)$ & 159 \\
\hline $\mathrm{C} 14-\mathrm{H} 14 \cdots \mathrm{O} 8 A^{\mathrm{i}}$ & 0.93 & 2.48 & $3.299(7)$ & 147 \\
\hline $\mathrm{C} 25-\mathrm{H} 25 \cdots \mathrm{O} 7^{\mathrm{ii}}$ & 0.93 & 2.51 & $3.391(4)$ & 158 \\
\hline $\mathrm{C} 25-\mathrm{H} 25 \cdots \mathrm{O} 7 A^{\mathrm{ii}}$ & 0.93 & 2.66 & $3.457(9)$ & 144 \\
\hline $\mathrm{C} 13-\mathrm{H} 13 \cdots \mathrm{O} 3^{\mathrm{iii}}$ & 0.93 & 2.60 & $3.372(3)$ & 141 \\
\hline $\mathrm{C} 23-\mathrm{H} 23 \cdots \mathrm{O} 5^{\mathrm{iv}}$ & 0.93 & 2.59 & $3.364(3)$ & 141 \\
\hline $\mathrm{O} 8-\mathrm{H} 8 A \cdots \mathrm{O} 2$ & 0.82 & 2.12 & $2.926(4)$ & 167 \\
\hline $\mathrm{O} 7-\mathrm{H} 7 A \cdots \mathrm{O} 6$ & 0.82 & 2.18 & $2.940(3)$ & 153 \\
\hline $\mathrm{O} 7-\mathrm{H} 7 A \cdots \mathrm{O} 4$ & 0.82 & 2.64 & $3.246(4)$ & 132 \\
\hline $\mathrm{O} 8 A-\mathrm{H} 8 A A \cdots \mathrm{O} 1$ & 0.82 & 2.18 & $2.984(6)$ & 166 \\
\hline
\end{tabular}

$x-\frac{1}{2},-y+\frac{1}{2}, z$.

Data collection: CrysAlis PRO (Agilent, 2013); cell refinement: CrysAlis PRO; data reduction: CrysAlis PRO; program(s) used to solve structure: SHELXS97 (Sheldrick, 2008); program(s) used to refine structure: SHELXL2014 (Sheldrick, 2008); molecular graphics: OLEX2 (Dolomanov et al., 2009); software used to prepare material for publication: $O L E X 2$.

\section{Acknowledgements}

We thank Professor A. N. Khlobystov for the sample of unsolvated $\left\{2,2^{\prime}\right.$-[ethylenebis(nitrilomethylidyne)]diphenolato- $\kappa^{4} O, N, N^{\prime}, O^{\prime}$ \}oxidovanadium(IV). We thank EPSRC (UK) for support.

Supporting information for this paper is available from the IUCr electronic archives (Reference: GG2143).

\section{References}

Agilent (2013). CrysAlis PRO. Agilent Technologies, Yarnton, England.

Dolomanov, O. V., Bourhis, L. J., Gildea, R. J., Howard, J. A. K. \& Puschmann, H. (2009). J. Appl. Cryst. 42, 339-341.

Flack, H. D. (1983). Acta Cryst. A39, 876-881.

Groom, C. R. \& Allen, F. H. (2014). Angew. Chem. Int. Ed. 53, 662-671.

Li, L. Z., Xu, T., Wang, D.-Q. \& Du, L.-Y. (2004). Huaxue Yanjiu Yu Yingyong (Chem. Res. Appl.), 16, 664-666.

Riley, P. E., Pecoraro, V. L., Carrano, C. J., Bonadies, J. A. \& Raymond, K. N. (1986). Inorg. Chem. 25, 154-160.

Sheldrick, G. M. (2008). Acta Cryst. A64, 112-122.

Wang, C., Yuan, J.-H., Xie, G., Yu, M.-J. \& Li, J. (2008). Acta Cryst. E64, m775$\mathrm{m} 776$. 


\section{supporting information}

Acta Cryst. (2014). E70, m380-m381 [doi:10.1107/S1600536814023332]

\section{Crystal structure of $\left\{2,2^{\prime}\right.$-[ethylenebis(nitrilomethanylylidene)]diphenolato- $\kappa^{4} O, N, N^{\prime}, O^{\prime}$ \}oxidovanadium(IV) methanol monosolvate}

\section{Rachel E. Hsuan, Jemma E. Hughes, Thomas H. Miller, Nabila Shaikh, Phoebe H. M.}

Cunningham, Alice E. O'Connor, Jeremiah P. Tidey and Alexander J. Blake

\section{S1. Experimental}

Crystals of the title complex were obtained by slow cooling of a methanolic solution of $\left\{2,2^{\prime}\right.$-[ethylenebis(nitrilomethylidyne)]diphenolato- $\kappa^{4} O, N, N^{\prime}, O^{\prime}$ \}oxidovanadium(IV).

\section{S2. Refinement}

With the exceptions of methyl groups (which were refined as rigid rotating groups) and $\mathrm{OH}$ groups (for which were idealized tetrahedral $\mathrm{OH}$ were refined as rigid rotating groups), $\mathrm{H}$ atoms were allowed to ride on their parent atoms with $U_{\text {iso }}(\mathrm{H})=1.5 \times U_{\text {eq }}(\mathrm{C}, \mathrm{O})$ for methyl and $\mathrm{OH}$ groups and $1.2 \times U_{\text {eq }}(\mathrm{C})$ for others.

The O8-C34, O8A-C34A, O7-C33 and O7A-C33A distances were restrained to a target value of 1.43 (1) $\AA$. SIMU and RIGU restraints (Sheldrick, 2008) were applied to the solvent molecules, and linked occupancies were used to stabilize the refinement of the $\mathrm{MeOH}$ solvent molecules.

For the absolute structure determination, the classical Flack method was preferred over Parsons because the s.u. was lower. 


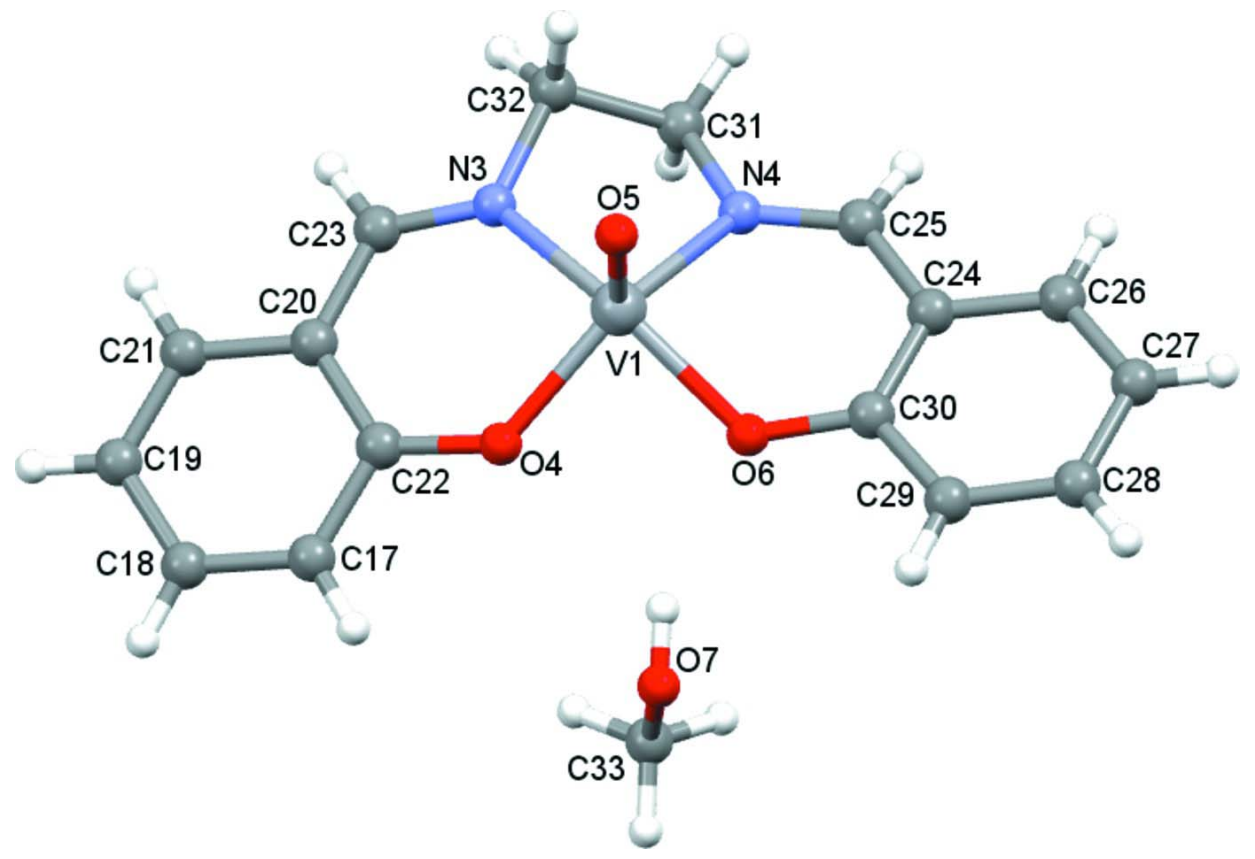

\section{Figure 1}

One of the two independent solvated molecules in the asymmetric unit, with atom labels and $50 \%$ probability displacement ellipsoids for non-H atoms. Only one component of the disordered $\mathrm{MeOH}$ molecule is shown.

\section{$\left\{2,2^{\prime}\right.$-[Ethylenebis(nitrilomethanylylidene)] diphenolato- $\left.\kappa^{4} O, N, N^{\prime}, O^{\prime}\right\}$ oxidovanadium(IV) methanol monosolvate}

\section{Crystal data}

$\left[\mathrm{V}\left(\mathrm{C}_{16} \mathrm{H}_{14} \mathrm{~N}_{2} \mathrm{O}_{2}\right) \mathrm{O}\right] \cdot \mathrm{CH}_{4} \mathrm{O}$

$M_{r}=365.27$

Orthorhombic, Pna2

$a=12.9597(4) \AA$

$b=8.8616(2) \AA$

$c=28.5426(7) \AA$

$V=3277.92(15) \AA^{3}$

$Z=8$

$F(000)=1512$

\section{Data collection}

\section{Agilent GV1000}

diffractometer with an Atlas CCD detector

Radiation source: Agilent GV1000 (Cu) X-ray

Source

Mirror monochromator

Detector resolution: 10.3271 pixels $\mathrm{mm}^{-1}$

$\omega$ scans

Absorption correction: gaussian

(CrysAlis PRO; Agilent, 2013)

\section{Refinement}

Refinement on $F^{2}$

Least-squares matrix: full

$R\left[F^{2}>2 \sigma\left(F^{2}\right)\right]=0.037$

$w R\left(F^{2}\right)=0.105$
$D_{\mathrm{x}}=1.480 \mathrm{Mg} \mathrm{m}^{-3}$

$\mathrm{Cu} K \alpha$ radiation, $\lambda=1.54184 \AA$

Cell parameters from 3840 reflections

$\theta=5.2-72.3^{\circ}$

$\mu=5.27 \mathrm{~mm}^{-1}$

$T=120 \mathrm{~K}$

Block, green

$0.31 \times 0.14 \times 0.05 \mathrm{~mm}$

$T_{\min }=0.480, T_{\max }=0.959$

7416 measured reflections

4634 independent reflections

4350 reflections with $I>2 \sigma(I)$

$R_{\text {int }}=0.025$

$\theta_{\max }=74.4^{\circ}, \theta_{\min }=5.2^{\circ}$

$h=-15 \rightarrow 15$

$k=-10 \rightarrow 6$

$l=-33 \rightarrow 34$

$S=1.04$

4634 reflections

479 parameters

32 restraints 
Primary atom site location: structure-invariant direct methods

Hydrogen site location: inferred from neighbouring sites

$\mathrm{H}$-atom parameters constrained

$w=1 /\left[\sigma^{2}\left(F_{\mathrm{o}}^{2}\right)+(0.065 P)^{2}+1.252 P\right]$

where $P=\left(F_{\mathrm{o}}^{2}+2 F_{\mathrm{c}}^{2}\right) / 3$
$(\Delta / \sigma)_{\max }=0.02$

$\Delta \rho_{\max }=0.42$ e $\AA^{-3}$

$\Delta \rho_{\min }=-0.29$ e $\AA^{-3}$

Absolute structure: Flack (1983), 1395 Friedel pairs

Absolute structure parameter: $0.066(9)$

Special details

Experimental. Absorption correction: CrysAlisPro version 1.171.36.32 (Agilent, 2013). Numerical absorption correction based on Gaussian integration over a multifaceted crystal model.

Geometry. All e.s.d.'s (except the e.s.d. in the dihedral angle between two 1.s. planes) are estimated using the full covariance matrix. The cell e.s.d.'s are taken into account individually in the estimation of e.s.d.'s in distances, angles and torsion angles; correlations between e.s.d.'s in cell parameters are only used when they are defined by crystal symmetry. An approximate (isotropic) treatment of cell e.s.d.'s is used for estimating e.s.d.'s involving 1.s. planes.

Fractional atomic coordinates and isotropic or equivalent isotropic displacement parameters $\left(\AA^{2}\right)$

\begin{tabular}{|c|c|c|c|c|c|}
\hline & $x$ & $y$ & $z$ & $U_{\text {iso }} * / U_{\text {eq }}$ & Occ. $(<1)$ \\
\hline V1 & 0.33525 & $1.13029(4)$ & $0.50170(2)$ & $0.02409(8)$ & \\
\hline $\mathrm{V} 2$ & 0.57988 & $0.36777(4)$ & $0.18892(2)$ & $0.02364(8)$ & \\
\hline $\mathrm{O} 1$ & $0.24854(12)$ & $0.95916(19)$ & $0.49027(6)$ & $0.0305(4)$ & \\
\hline $\mathrm{O} 2$ & $0.41375(12)$ & $1.06657(19)$ & $0.44719(6)$ & $0.0277(4)$ & \\
\hline $\mathrm{O} 3$ & $0.41104(12)$ & $1.1086(2)$ & $0.54575(6)$ & 0.0298 & \\
\hline $\mathrm{O} 4$ & $0.49307(12)$ & $0.53856(17)$ & $0.20089(6)$ & $0.0292(4)$ & \\
\hline O5 & $0.65555(12)$ & $0.39010(19)$ & $0.14477(6)$ & $0.0292(4)$ & \\
\hline O6 & $0.65825(12)$ & $0.4296(2)$ & $0.24318(6)$ & $0.0294(4)$ & \\
\hline N2 & $0.35312(15)$ & $1.3476(2)$ & $0.47830(7)$ & $0.0275(4)$ & \\
\hline N4 & $0.59693(16)$ & $0.1492(2)$ & $0.21225(7)$ & $0.0289(5)$ & \\
\hline C12 & $0.49325(19)$ & 1.1394 & $0.42862(7)$ & $0.0283(6)$ & \\
\hline C14 & $0.42784(18)$ & 1.3949 & $0.45247(8)$ & $0.0295(5)$ & \\
\hline H14 & 0.4327 & 1.4981 & 0.4471 & $0.035^{*}$ & \\
\hline $\mathrm{C} 30$ & $0.73712(18)$ & $0.3563(3)$ & $0.26195(8)$ & $0.0306(6)$ & \\
\hline $\mathrm{C} 3$ & $0.15814(17)$ & $0.9193(3)$ & $0.50810(8)$ & $0.0280(5)$ & \\
\hline $\mathrm{C} 25$ & $0.6714(2)$ & $0.1024(3)$ & $0.23826(8)$ & $0.0322(6)$ & \\
\hline $\mathrm{H} 25$ & 0.6761 & -0.0010 & 0.2435 & $0.039 *$ & \\
\hline N1 & $0.20834(14)$ & $1.2335(2)$ & $0.53104(7)$ & $0.0269(4)$ & \\
\hline $\mathrm{C} 26$ & 0.8334 & 0.1282 & $0.28130(9)$ & $0.0409(7)$ & \\
\hline $\mathrm{H} 26$ & 0.8397 & 0.0237 & 0.2803 & $0.049^{*}$ & \\
\hline N3 & $0.45275(15)$ & $0.2651(2)$ & $0.15943(7)$ & 0.0278 & \\
\hline $\mathrm{C} 4$ & $0.09719(17)$ & $1.0158(3)$ & $0.53631(7)$ & $0.0273(5)$ & \\
\hline $\mathrm{C} 10$ & $0.6518(2)$ & $1.1282(4)$ & $0.38350(9)$ & $0.0423(8)$ & \\
\hline H10 & 0.7005 & 1.0710 & 0.3674 & $0.051^{*}$ & \\
\hline $\mathrm{C} 8$ & 0.5908 & 1.3668 & $0.40987(8)$ & $0.0396(7)$ & \\
\hline H8 & 0.5986 & 1.4710 & 0.4113 & $0.048^{*}$ & \\
\hline $\mathrm{C} 15$ & $0.2266(2)$ & $1.3957(3)$ & $0.53910(9)$ & $0.0326(6)$ & \\
\hline H15A & 0.2729 & 1.4102 & 0.5654 & $0.039 *$ & \\
\hline H15B & 0.1621 & 1.4470 & 0.5457 & $0.039 *$ & \\
\hline $\mathrm{C} 22$ & $0.40338(16)$ & 0.5789 & $0.18283(8)$ & $0.0273(5)$ & \\
\hline $\mathrm{C} 17$ & $0.36417(19)$ & 0.7228 & $0.19338(10)$ & $0.0352(6)$ & \\
\hline
\end{tabular}




\begin{tabular}{|c|c|c|c|c|c|}
\hline H17 & 0.4014 & 0.7860 & 0.2132 & $0.042 *$ & \\
\hline $\mathrm{C} 24$ & 0.74815 (18) & $0.1970(3)$ & $0.26002(8)$ & $0.0316(6)$ & \\
\hline $\mathrm{C} 13$ & $0.12347(18)$ & $1.1718(3)$ & $0.54445(8)$ & $0.0295(5)$ & \\
\hline $\mathrm{H} 13$ & 0.0761 & 1.2316 & 0.5604 & $0.035^{*}$ & \\
\hline $\mathrm{C} 18$ & $0.2719(2)$ & $0.7725(3)$ & $0.17509(9)$ & $0.0390(6)$ & \\
\hline $\mathrm{H} 18$ & 0.2478 & 0.8680 & 0.1830 & $0.047^{*}$ & \\
\hline $\mathrm{C} 1$ & $0.0269(2)$ & $0.7261(3)$ & $0.51707(9)$ & $0.0375(6)$ & \\
\hline $\mathrm{H} 1$ & 0.0032 & 0.6295 & 0.5102 & $0.045^{*}$ & \\
\hline $\mathrm{C} 9$ & $0.50432(18)$ & $1.2990(3)$ & $0.43126(7)$ & $0.0301(6)$ & \\
\hline $\mathrm{C} 7$ & $0.6641(2)$ & $1.2817(4)$ & $0.38686(9)$ & $0.0451(8)$ & \\
\hline $\mathrm{H} 7$ & 0.7215 & 1.3281 & 0.3737 & $0.054^{*}$ & \\
\hline $\mathrm{C} 20$ & $0.34189(17)$ & 0.4834 (3) & $0.15433(7)$ & $0.0278(5)$ & \\
\hline $\mathrm{C} 28$ & $0.8976(2)$ & $0.3678(4)$ & $0.30590(9)$ & $0.0440(8)$ & \\
\hline $\mathrm{H} 28$ & 0.9476 & 0.4251 & 0.3211 & $0.053^{*}$ & \\
\hline $\mathrm{C} 2$ & $0.11882(19)$ & $0.7752(3)$ & $0.49797(9)$ & $0.0331(6)$ & \\
\hline $\mathrm{H} 2$ & 0.1554 & 0.7117 & 0.4780 & $0.040^{*}$ & \\
\hline $\mathrm{C} 23$ & $0.36811(17)$ & $0.3277(3)$ & $0.14619(8)$ & $0.0283(5)$ & \\
\hline $\mathrm{H} 23$ & 0.3205 & 0.2683 & 0.1302 & $0.034^{*}$ & \\
\hline $\mathrm{C} 16$ & $0.27463(19)$ & $1.4566(3)$ & $0.49437(9)$ & $0.0333(6)$ & \\
\hline H16A & 0.2220 & 1.4695 & 0.4705 & $0.040^{*}$ & \\
\hline H16B & 0.3064 & 1.5539 & 0.5003 & $0.040^{*}$ & \\
\hline $\mathrm{C} 5$ & $0.00379(18)$ & $0.9625(3)$ & $0.55506(8)$ & $0.0323(6)$ & \\
\hline H5 & -0.0355 & 1.0263 & 0.5738 & $0.039^{*}$ & \\
\hline $\mathrm{C} 21$ & $0.24831(18)$ & $0.5379(3)$ & $0.13563(8)$ & $0.0334(6)$ & \\
\hline $\mathrm{H} 21$ & 0.2088 & 0.4753 & 0.1166 & $0.040^{*}$ & \\
\hline $\mathrm{C} 11$ & 0.56715 (19) & $1.0573(3)$ & $0.40398(8)$ & $0.0344(6)$ & \\
\hline H11 & 0.5598 & 0.9533 & 0.4011 & $0.041^{*}$ & \\
\hline $\mathrm{C} 19$ & $0.21417(19)$ & $0.6823(3)$ & $0.14501(9)$ & $0.0377(6)$ & \\
\hline H19 & 0.1538 & 0.7186 & 0.1315 & $0.045^{*}$ & \\
\hline $\mathrm{C} 27$ & $0.9082(2)$ & $0.2117(4)$ & $0.30372(9)$ & $0.0508(8)$ & \\
\hline $\mathrm{H} 27$ & 0.9650 & 0.1644 & 0.3172 & $0.061^{*}$ & \\
\hline C6 & -0.03109 (19) & $0.8186(3)$ & $0.54647(9)$ & $0.0362(6)$ & \\
\hline H6 & -0.0919 & 0.7839 & 0.5600 & $0.043 *$ & \\
\hline $\mathrm{C} 29$ & $0.81364(18)$ & $0.4385(4)$ & $0.28575(8)$ & $0.0360(6)$ & \\
\hline H29 & 0.8076 & 0.5429 & 0.2880 & $0.043^{*}$ & \\
\hline $\mathrm{C} 31$ & $0.51874(19)$ & $0.0415(3)$ & $0.19582(9)$ & $0.0328(6)$ & \\
\hline $\mathrm{H} 31 \mathrm{~A}$ & 0.4662 & 0.0279 & 0.2196 & $0.039 *$ & \\
\hline H31B & 0.5505 & -0.0555 & 0.1896 & $0.039^{*}$ & \\
\hline $\mathrm{C} 32$ & $0.4706(2)$ & $0.1028(3)$ & $0.15151(9)$ & $0.0332(6)$ & \\
\hline $\mathrm{H} 32 \mathrm{~A}$ & 0.5165 & 0.0879 & 0.1251 & $0.040^{*}$ & \\
\hline H32B & 0.4059 & 0.0518 & 0.1451 & $0.040^{*}$ & \\
\hline $\mathrm{O} 8$ & $0.3881(3)$ & $0.7530(4)$ & $0.41726(11)$ & $0.0533(9)$ & $0.678(4)$ \\
\hline $\mathrm{H} 8 \mathrm{~A}$ & 0.3953 & 0.8354 & 0.4299 & $0.080^{*}$ & $0.678(4)$ \\
\hline O7 & $0.6257(3)$ & $0.7431(3)$ & $0.27352(11)$ & $0.0573(9)$ & $0.750(5)$ \\
\hline H7A & 0.6295 & 0.6685 & 0.2566 & $0.086^{*}$ & $0.750(5)$ \\
\hline $\mathrm{C} 33$ & $0.5510(3)$ & $0.7185(6)$ & $0.30846(13)$ & $0.0506(11)$ & $0.750(5)$ \\
\hline H33A & 0.4851 & 0.7011 & 0.2940 & $0.076^{*}$ & $0.750(5)$ \\
\hline H33B & 0.5469 & 0.8056 & 0.3284 & $0.076^{*}$ & 0.750 \\
\hline
\end{tabular}


supporting information

$\begin{array}{llllll}\text { H33C } & 0.5700 & 0.6320 & 0.3268 & 0.076^{*} & 0.750(5) \\ \text { C34 } & 0.3009(4) & 0.7570(6) & 0.38743(15) & 0.0614(14) & 0.678(4) \\ \text { H34A } & 0.3138 & 0.6966 & 0.3602 & 0.092^{*} & 0.678(4) \\ \text { H34B } & 0.2876 & 0.8593 & 0.3781 & 0.092^{*} & 0.678(4) \\ \text { H34C } & 0.2419 & 0.7180 & 0.4038 & 0.092^{*} & 0.678(4) \\ \text { O7A } & 0.5715(10) & 0.7560(9) & 0.2706(2) & 0.068(3) & 0.250(5) \\ \text { H7AA } & 0.5107 & 0.7627 & 0.2629 & 0.103^{*} & 0.250(5) \\ \text { C33A } & 0.5784(9) & 0.6910(16) & 0.3163(3) & 0.073(4) & 0.250(5) \\ \text { H33D } & 0.6391 & 0.6293 & 0.3182 & 0.110^{*} & 0.250(5) \\ \text { H33E } & 0.5185 & 0.6302 & 0.3221 & 0.110^{*} & 0.250(5) \\ \text { H33F } & 0.5823 & 0.7700 & 0.3393 & 0.110^{*} & 0.250(5) \\ \text { C34A } & 0.3143(7) & 0.7847(9) & 0.37978(19) & 0.041(2) & 0.322(4) \\ \text { H34D } & 0.3315 & 0.7086 & 0.3572 & 0.061^{*} & 0.322(4) \\ \text { H34E } & 0.3511 & 0.8759 & 0.3726 & 0.061^{*} & 0.322(4) \\ \text { H34F } & 0.2414 & 0.8038 & 0.3788 & 0.061^{*} & 0.322(4) \\ \text { O8A } & 0.3422(6) & 0.7342(7) & 0.42510(16) & 0.0481(19) & 0.322(4) \\ \text { H8AA } & 0.3271 & 0.7990 & 0.4444 & 0.072^{*} & 0.322(4)\end{array}$

Atomic displacement parameters $\left(\AA^{2}\right)$

\begin{tabular}{lllllll}
\hline & $U^{11}$ & $U^{22}$ & $U^{33}$ & $U^{12}$ & $U^{13}$ & $U^{23}$ \\
\hline $\mathrm{V} 1$ & $0.02276(15)$ & $0.02190(17)$ & $0.02763(14)$ & $0.00204(14)$ & $-0.00088(14)$ & $-0.00101(14)$ \\
$\mathrm{V} 2$ & $0.02314(15)$ & $0.02248(17)$ & $0.02531(14)$ & $-0.00205(14)$ & $0.00061(14)$ & $-0.00063(14)$ \\
$\mathrm{O} 1$ & $0.0274(7)$ & $0.0247(8)$ & $0.0394(8)$ & $0.0009(6)$ & $0.0042(7)$ & $-0.0034(7)$ \\
$\mathrm{O} 2$ & $0.0263(7)$ & $0.0274(8)$ & $0.0295(7)$ & $-0.0023(7)$ & $0.0018(6)$ & $-0.0025(6)$ \\
$\mathrm{O} 3$ & $0.0232(7)$ & $0.0338(9)$ & $0.0325(7)$ & $0.0038(7)$ & $-0.0038(6)$ & $0.0015(7)$ \\
O4 & $0.0276(7)$ & $0.0246(8)$ & $0.0355(7)$ & $-0.0019(6)$ & $-0.0030(7)$ & $-0.0032(7)$ \\
O5 & $0.0249(7)$ & $0.0318(8)$ & $0.0310(7)$ & $-0.0031(7)$ & $0.0021(6)$ & $0.0000(7)$ \\
O6 & $0.0282(7)$ & $0.0312(8)$ & $0.0289(6)$ & $-0.0007(7)$ & $-0.0021(6)$ & $-0.0023(7)$ \\
N2 & $0.0288(8)$ & $0.0239(9)$ & $0.0297(8)$ & $0.0006(8)$ & $-0.0091(8)$ & $-0.0023(8)$ \\
N4 & $0.0326(9)$ & $0.0250(9)$ & $0.0290(8)$ & $0.0013(8)$ & $0.0067(8)$ & $0.0004(8)$ \\
C12 & $0.0333(11)$ & $0.0334(12)$ & $0.0183(8)$ & $-0.0018(10)$ & $-0.0057(9)$ & $0.0025(8)$ \\
C14 & $0.0329(11)$ & $0.0300(11)$ & $0.0255(9)$ & $-0.0055(10)$ & $-0.0071(9)$ & $0.0028(9)$ \\
C30 & $0.0270(10)$ & $0.0426(13)$ & $0.0222(8)$ & $0.0018(10)$ & $0.0061(9)$ & $-0.0022(9)$ \\
C3 & $0.0259(9)$ & $0.0266(10)$ & $0.0316(9)$ & $0.0010(9)$ & $-0.0035(9)$ & $0.0032(9)$ \\
C25 & $0.0432(12)$ & $0.0287(11)$ & $0.0248(9)$ & $0.0079(10)$ & $0.0086(10)$ & $0.0029(9)$ \\
N1 & $0.0230(8)$ & $0.0278(9)$ & $0.0301(8)$ & $0.0034(7)$ & $-0.0022(7)$ & $-0.0059(8)$ \\
C26 & $0.0420(13)$ & $0.0542(16)$ & $0.0266(10)$ & $0.0179(12)$ & $0.0028(11)$ & $0.0024(11)$ \\
N3 & $0.0285(9)$ & $0.0256(9)$ & $0.0294(8)$ & $-0.0043(8)$ & $0.0036(8)$ & $-0.0056(8)$ \\
C4 & $0.0263(10)$ & $0.0307(11)$ & $0.0248(8)$ & $0.0021(9)$ & $-0.0051(8)$ & $0.0022(9)$ \\
C10 & $0.0376(13)$ & $0.0658(19)$ & $0.0234(9)$ & $0.0038(13)$ & $0.0046(10)$ & $0.0017(11)$ \\
C8 & $0.0449(14)$ & $0.0480(15)$ & $0.0260(10)$ & $-0.0143(12)$ & $-0.0023(10)$ & $0.0045(10)$ \\
C15 & $0.0321(11)$ & $0.0289(11)$ & $0.0368(11)$ & $0.0081(10)$ & $-0.0023(10)$ & $-0.0079(9)$ \\
C22 & $0.0228(9)$ & $0.0292(11)$ & $0.0300(9)$ & $0.0006(9)$ & $0.0034(9)$ & $0.0061(9)$ \\
C17 & $0.0319(10)$ & $0.0290(11)$ & $0.0447(12)$ & $-0.0012(10)$ & $0.0053(11)$ & $0.0022(11)$ \\
C24 & $0.0346(11)$ & $0.0369(12)$ & $0.0233(8)$ & $0.0073(11)$ & $0.0044(9)$ & $0.0012(10)$ \\
C13 & $0.0254(10)$ & $0.0372(12)$ & $0.0257(9)$ & $0.0077(10)$ & $-0.0029(9)$ & $-0.0041(9)$ \\
C18 & $0.0391(12)$ & $0.0319(12)$ & $0.0458(12)$ & $0.0081(11)$ & $0.0133(11)$ & $0.0098(11)$ \\
& & & & &
\end{tabular}


supporting information

\begin{tabular}{lllllll} 
C1 & $0.0357(12)$ & $0.0302(11)$ & $0.0468(12)$ & $-0.0025(11)$ & $-0.0094(11)$ & $0.0112(11)$ \\
C9 & $0.0310(10)$ & $0.0420(13)$ & $0.0172(8)$ & $-0.0051(11)$ & $-0.0043(9)$ & $0.0023(9)$ \\
C7 & $0.0394(13)$ & $0.0687(19)$ & $0.0272(10)$ & $-0.0097(13)$ & $0.0092(10)$ & $0.0072(12)$ \\
C20 & $0.0225(9)$ & $0.0351(12)$ & $0.0258(9)$ & $-0.0038(9)$ & $0.0030(8)$ & $-0.0002(9)$ \\
C28 & $0.0322(12)$ & $0.075(2)$ & $0.0250(10)$ & $-0.0029(13)$ & $-0.0005(10)$ & $-0.0022(11)$ \\
C2 & $0.0306(11)$ & $0.0276(11)$ & $0.0411(11)$ & $-0.0002(10)$ & $-0.0026(10)$ & $0.0023(10)$ \\
C23 & $0.0230(9)$ & $0.0349(12)$ & $0.0269(9)$ & $-0.0054(10)$ & $0.0001(8)$ & $-0.0029(9)$ \\
C16 & $0.0349(11)$ & $0.0237(11)$ & $0.0412(11)$ & $0.0045(10)$ & $-0.0115(10)$ & $-0.0012(9)$ \\
C5 & $0.0268(10)$ & $0.0438(14)$ & $0.0262(9)$ & $0.0024(10)$ & $-0.0019(9)$ & $0.0028(10)$ \\
C21 & $0.0265(10)$ & $0.0468(14)$ & $0.0269(9)$ & $-0.0018(11)$ & $0.0017(9)$ & $0.0081(10)$ \\
C11 & $0.0348(11)$ & $0.0461(14)$ & $0.0223(8)$ & $0.0024(11)$ & $-0.0023(9)$ & $-0.0005(10)$ \\
C19 & $0.0292(11)$ & $0.0481(14)$ & $0.0358(10)$ & $0.0045(11)$ & $0.0040(10)$ & $0.0134(11)$ \\
C27 & $0.0431(13)$ & $0.079(2)$ & $0.0301(11)$ & $0.0202(15)$ & $-0.0037(11)$ & $0.0057(13)$ \\
C6 & $0.0263(10)$ & $0.0466(14)$ & $0.0356(10)$ & $-0.0044(11)$ & $-0.0024(10)$ & $0.0126(11)$ \\
C29 & $0.0291(10)$ & $0.0553(15)$ & $0.0238(9)$ & $-0.0055(11)$ & $0.0001(9)$ & $-0.0019(10)$ \\
C31 & $0.0377(11)$ & $0.0225(11)$ & $0.0383(11)$ & $-0.0061(9)$ & $0.0077(10)$ & $-0.0031(10)$ \\
C32 & $0.0340(11)$ & $0.0263(11)$ & $0.0394(11)$ & $-0.0039(10)$ & $0.0084(10)$ & $-0.0086(10)$ \\
O8 & $0.0693(19)$ & $0.0407(16)$ & $0.0501(15)$ & $0.0048(15)$ & $-0.0044(16)$ & $-0.0062(14)$ \\
O7 & $0.0792(19)$ & $0.0308(13)$ & $0.0618(16)$ & $-0.0094(14)$ & $0.0178(16)$ & $-0.0105(13)$ \\
C33 & $0.055(2)$ & $0.058(2)$ & $0.0383(16)$ & $0.006(2)$ & $-0.0047(17)$ & $-0.0122(16)$ \\
C34 & $0.086(3)$ & $0.057(3)$ & $0.0412(19)$ & $-0.025(2)$ & $-0.008(2)$ & $0.0187(19)$ \\
O7A & $0.122(8)$ & $0.028(4)$ & $0.055(4)$ & $0.017(5)$ & $-0.020(4)$ & $-0.016(3)$ \\
C33A & $0.043(5)$ & $0.105(9)$ & $0.071(5)$ & $0.049(5)$ & $-0.002(4)$ & $0.009(5)$ \\
C34A & $0.076(5)$ & $0.028(4)$ & $0.019(3)$ & $-0.025(4)$ & $0.012(3)$ & $0.000(3)$ \\
O8A & $0.090(5)$ & $0.029(3)$ & $0.025(2)$ & $0.008(3)$ & $-0.002(3)$ & $-0.003(2)$ \\
& & & & & & \\
\hline
\end{tabular}

Geometric parameters $\left(\AA,{ }^{\circ}\right)$

\begin{tabular}{llll}
\hline $\mathrm{V} 1-\mathrm{O} 1$ & $1.9155(17)$ & $\mathrm{C} 13-\mathrm{H} 13$ & 0.9300 \\
$\mathrm{~V} 1-\mathrm{O} 2$ & $1.9429(16)$ & $\mathrm{C} 18-\mathrm{H} 18$ & 0.9300 \\
$\mathrm{~V} 1-\mathrm{O} 3$ & $1.6070(17)$ & $\mathrm{C} 18-\mathrm{C} 19$ & $1.392(4)$ \\
$\mathrm{V} 1-\mathrm{N} 1$ & $2.0597(19)$ & $\mathrm{C} 1-\mathrm{H} 1$ & 0.9300 \\
$\mathrm{~V} 1-\mathrm{N} 2$ & $2.051(2)$ & $\mathrm{C} 1-\mathrm{C} 2$ & $1.381(4)$ \\
$\mathrm{V} 2-\mathrm{O} 4$ & $1.9164(16)$ & $\mathrm{C} 1-\mathrm{C} 6$ & $1.393(4)$ \\
$\mathrm{V} 2-\mathrm{O} 5$ & $1.6089(16)$ & $\mathrm{C} 7-\mathrm{H} 7$ & 0.9300 \\
$\mathrm{~V} 2-\mathrm{O} 6$ & $1.9314(16)$ & $\mathrm{C} 20-\mathrm{C} 23$ & $1.440(4)$ \\
$\mathrm{V} 2-\mathrm{N} 3$ & $2.062(2)$ & $\mathrm{C} 20-\mathrm{C} 21$ & $1.410(3)$ \\
$\mathrm{V} 2-\mathrm{N} 4$ & $2.060(2)$ & $\mathrm{C} 28-\mathrm{H} 28$ & 0.9300 \\
$\mathrm{O} 6-\mathrm{C} 30$ & $1.324(3)$ & $\mathrm{C} 28-\mathrm{C} 27$ & $1.392(5)$ \\
$\mathrm{O} 2-\mathrm{C} 12$ & $1.326(3)$ & $\mathrm{C} 28-\mathrm{C} 29$ & $1.381(4)$ \\
$\mathrm{N} 4-\mathrm{C} 25$ & $1.286(3)$ & $\mathrm{C} 2-\mathrm{H} 2$ & 0.9300 \\
$\mathrm{~N} 4-\mathrm{C} 31$ & $\mathrm{C} 23-\mathrm{H} 23$ & 0.9300 \\
$\mathrm{~N} 2-\mathrm{C} 14$ & $1.469(3)$ & $\mathrm{C} 16-\mathrm{H} 16 \mathrm{~A}$ & 0.9700 \\
$\mathrm{~N} 2-\mathrm{C} 16$ & $1.287(3)$ & $\mathrm{C} 16-\mathrm{H} 16 \mathrm{~B}$ & 0.9700 \\
$\mathrm{O} 1-\mathrm{C} 3$ & $1.476(3)$ & $\mathrm{C} 5-\mathrm{H} 5$ & 0.9300 \\
$\mathrm{C} 12-\mathrm{C} 9$ & $1.325(3)$ & $\mathrm{C} 5-\mathrm{C} 6$ & $1.375(4)$ \\
$\mathrm{C} 12-\mathrm{C} 11$ & $1.424(4)$ & $\mathrm{C} 21-\mathrm{H} 21$ & 0.9300 \\
$\mathrm{O} 4-\mathrm{C} 22$ & $1.393(4)$ & $\mathrm{C} 21-\mathrm{C} 19$ & $1.380(4)$
\end{tabular}




\begin{tabular}{|c|c|c|c|}
\hline C14-H14 & 0.9300 & $\mathrm{C} 11-\mathrm{H} 11$ & 0.9300 \\
\hline $\mathrm{C} 14-\mathrm{C} 9$ & $1.439(3)$ & C19-H19 & 0.9300 \\
\hline $\mathrm{C} 30-\mathrm{C} 24$ & $1.420(4)$ & $\mathrm{C} 27-\mathrm{H} 27$ & 0.9300 \\
\hline $\mathrm{C} 30-\mathrm{C} 29$ & $1.406(4)$ & $\mathrm{C} 6-\mathrm{H} 6$ & 0.9300 \\
\hline $\mathrm{C} 3-\mathrm{C} 4$ & $1.415(3)$ & $\mathrm{C} 29-\mathrm{H} 29$ & 0.9300 \\
\hline $\mathrm{C} 3-\mathrm{C} 2$ & $1.405(4)$ & $\mathrm{C} 31-\mathrm{H} 31 \mathrm{~A}$ & 0.9700 \\
\hline $\mathrm{C} 25-\mathrm{H} 25$ & 0.9300 & $\mathrm{C} 31-\mathrm{H} 31 \mathrm{~B}$ & 0.9700 \\
\hline $\mathrm{C} 25-\mathrm{C} 24$ & $1.442(4)$ & $\mathrm{C} 31-\mathrm{C} 32$ & $1.511(4)$ \\
\hline $\mathrm{N} 1-\mathrm{C} 15$ & $1.474(3)$ & $\mathrm{C} 32-\mathrm{H} 32 \mathrm{~A}$ & 0.9700 \\
\hline $\mathrm{N} 1-\mathrm{C} 13$ & $1.286(3)$ & $\mathrm{C} 32-\mathrm{H} 32 \mathrm{~B}$ & 0.9700 \\
\hline $\mathrm{C} 26-\mathrm{H} 26$ & 0.9300 & $\mathrm{O} 8-\mathrm{H} 8 \mathrm{~A}$ & 0.8200 \\
\hline $\mathrm{C} 26-\mathrm{C} 24$ & $1.401(4)$ & $\mathrm{O} 8-\mathrm{C} 34$ & $1.416(6)$ \\
\hline $\mathrm{C} 26-\mathrm{C} 27$ & $1.377(4)$ & $\mathrm{O} 7-\mathrm{H} 7 \mathrm{~A}$ & 0.8200 \\
\hline $\mathrm{N} 3-\mathrm{C} 23$ & $1.286(3)$ & $\mathrm{O} 7-\mathrm{C} 33$ & $1.407(5)$ \\
\hline $\mathrm{N} 3-\mathrm{C} 32$ & $1.474(3)$ & $\mathrm{C} 33-\mathrm{H} 33 \mathrm{~A}$ & 0.9600 \\
\hline $\mathrm{C} 4-\mathrm{C} 13$ & $1.443(4)$ & $\mathrm{C} 33-\mathrm{H} 33 \mathrm{~B}$ & 0.9600 \\
\hline $\mathrm{C} 4-\mathrm{C} 5$ & $1.405(3)$ & $\mathrm{C} 33-\mathrm{H} 33 \mathrm{C}$ & 0.9600 \\
\hline $\mathrm{C} 10-\mathrm{H} 10$ & 0.9300 & $\mathrm{C} 34-\mathrm{H} 34 \mathrm{~A}$ & 0.9600 \\
\hline $\mathrm{C} 10-\mathrm{C} 7$ & $1.373(5)$ & $\mathrm{C} 34-\mathrm{H} 34 \mathrm{~B}$ & 0.9600 \\
\hline $\mathrm{C} 10-\mathrm{C} 11$ & $1.393(4)$ & $\mathrm{C} 34-\mathrm{H} 34 \mathrm{C}$ & 0.9600 \\
\hline $\mathrm{C} 8-\mathrm{H} 8$ & 0.9300 & $\mathrm{O} 7 \mathrm{~A}-\mathrm{H} 7 \mathrm{AA}$ & 0.8200 \\
\hline $\mathrm{C} 8-\mathrm{C} 9$ & $1.411(4)$ & $\mathrm{O} 7 \mathrm{~A}-\mathrm{C} 33 \mathrm{~A}$ & $1.428(9)$ \\
\hline $\mathrm{C} 8-\mathrm{C} 7$ & $1.379(4)$ & $\mathrm{C} 33 \mathrm{~A}-\mathrm{H} 33 \mathrm{D}$ & 0.9600 \\
\hline C15-H15A & 0.9700 & $\mathrm{C} 33 \mathrm{~A}-\mathrm{H} 33 \mathrm{E}$ & 0.9600 \\
\hline $\mathrm{C} 15-\mathrm{H} 15 \mathrm{~B}$ & 0.9700 & $\mathrm{C} 33 \mathrm{~A}-\mathrm{H} 33 \mathrm{~F}$ & 0.9600 \\
\hline $\mathrm{C} 15-\mathrm{C} 16$ & $1.520(4)$ & C34A-H34D & 0.9600 \\
\hline $\mathrm{C} 22-\mathrm{C} 17$ & $1.405(4)$ & $\mathrm{C} 34 \mathrm{~A}-\mathrm{H} 34 \mathrm{E}$ & 0.9600 \\
\hline $\mathrm{C} 22-\mathrm{C} 20$ & $1.419(3)$ & $\mathrm{C} 34 \mathrm{~A}-\mathrm{H} 34 \mathrm{~F}$ & 0.9600 \\
\hline C17-H17 & 0.9300 & $\mathrm{C} 34 \mathrm{~A}-\mathrm{O} 8 \mathrm{~A}$ & $1.416(7)$ \\
\hline $\mathrm{C} 17-\mathrm{C} 18$ & $1.376(4)$ & O8A-H8AA & 0.8200 \\
\hline $\mathrm{O} 2-\mathrm{V} 1-\mathrm{N} 2$ & $87.30(8)$ & $\mathrm{C} 6-\mathrm{C} 1-\mathrm{H} 1$ & 119.4 \\
\hline $\mathrm{O} 2-\mathrm{V} 1-\mathrm{N} 1$ & $150.78(7)$ & $\mathrm{C} 12-\mathrm{C} 9-\mathrm{C} 14$ & $122.6(2)$ \\
\hline $\mathrm{O} 3-\mathrm{V} 1-\mathrm{O} 2$ & $105.76(8)$ & $\mathrm{C} 8-\mathrm{C} 9-\mathrm{C} 12$ & $118.7(2)$ \\
\hline $\mathrm{O} 3-\mathrm{V} 1-\mathrm{N} 2$ & $107.35(9)$ & $\mathrm{C} 8-\mathrm{C} 9-\mathrm{C} 14$ & $118.6(2)$ \\
\hline $\mathrm{O} 3-\mathrm{V} 1-\mathrm{O} 1$ & $113.39(8)$ & $\mathrm{C} 10-\mathrm{C} 7-\mathrm{C} 8$ & $119.7(3)$ \\
\hline $\mathrm{O} 3-\mathrm{V} 1-\mathrm{N} 1$ & $102.90(8)$ & $\mathrm{C} 10-\mathrm{C} 7-\mathrm{H} 7$ & 120.2 \\
\hline $\mathrm{N} 2-\mathrm{V} 1-\mathrm{N} 1$ & $78.80(8)$ & $\mathrm{C} 8-\mathrm{C} 7-\mathrm{H} 7$ & 120.2 \\
\hline $\mathrm{O} 1-\mathrm{V} 1-\mathrm{O} 2$ & $86.60(7)$ & $\mathrm{C} 22-\mathrm{C} 20-\mathrm{C} 23$ & $122.1(2)$ \\
\hline $\mathrm{O} 1-\mathrm{V} 1-\mathrm{N} 2$ & $138.94(8)$ & $\mathrm{C} 21-\mathrm{C} 20-\mathrm{C} 22$ & $119.7(2)$ \\
\hline $\mathrm{O} 1-\mathrm{V} 1-\mathrm{N} 1$ & $87.26(8)$ & $\mathrm{C} 21-\mathrm{C} 20-\mathrm{C} 23$ & $118.1(2)$ \\
\hline $\mathrm{O} 6-\mathrm{V} 2-\mathrm{N} 4$ & $87.20(8)$ & $\mathrm{C} 27-\mathrm{C} 28-\mathrm{H} 28$ & 119.6 \\
\hline $\mathrm{O} 6-\mathrm{V} 2-\mathrm{N} 3$ & $150.79(7)$ & $\mathrm{C} 29-\mathrm{C} 28-\mathrm{H} 28$ & 119.6 \\
\hline $\mathrm{O} 5-\mathrm{V} 2-\mathrm{O} 6$ & $105.81(8)$ & $\mathrm{C} 29-\mathrm{C} 28-\mathrm{C} 27$ & $120.7(3)$ \\
\hline $\mathrm{O} 5-\mathrm{V} 2-\mathrm{N} 4$ & $107.66(8)$ & $\mathrm{C} 3-\mathrm{C} 2-\mathrm{H} 2$ & 119.4 \\
\hline $\mathrm{O} 5-\mathrm{V} 2-\mathrm{O} 4$ & $113.60(8)$ & $\mathrm{C} 1-\mathrm{C} 2-\mathrm{C} 3$ & $121.2(2)$ \\
\hline $\mathrm{O} 5-\mathrm{V} 2-\mathrm{N} 3$ & $102.81(8)$ & $\mathrm{C} 1-\mathrm{C} 2-\mathrm{H} 2$ & 119.4 \\
\hline $\mathrm{N} 4-\mathrm{V} 2-\mathrm{N} 3$ & $78.63(8)$ & $\mathrm{N} 3-\mathrm{C} 23-\mathrm{C} 20$ & $124.6(2)$ \\
\hline
\end{tabular}




\begin{tabular}{|c|c|c|c|}
\hline $\mathrm{O} 4-\mathrm{V} 2-\mathrm{O} 6$ & $86.66(7)$ & $\mathrm{N} 3-\mathrm{C} 23-\mathrm{H} 23$ & 117.7 \\
\hline $\mathrm{O} 4-\mathrm{V} 2-\mathrm{N} 4$ & $138.40(8)$ & $\mathrm{C} 20-\mathrm{C} 23-\mathrm{H} 23$ & 117.7 \\
\hline $\mathrm{O} 4-\mathrm{V} 2-\mathrm{N} 3$ & $87.25(7)$ & $\mathrm{N} 2-\mathrm{C} 16-\mathrm{C} 15$ & $108.1(2)$ \\
\hline $\mathrm{C} 30-\mathrm{O} 6-\mathrm{V} 2$ & $126.23(15)$ & $\mathrm{N} 2-\mathrm{C} 16-\mathrm{H} 16 \mathrm{~A}$ & 110.1 \\
\hline $\mathrm{C} 12-\mathrm{O} 2-\mathrm{V} 1$ & $125.84(15)$ & $\mathrm{N} 2-\mathrm{C} 16-\mathrm{H} 16 \mathrm{~B}$ & 110.1 \\
\hline $\mathrm{C} 25-\mathrm{N} 4-\mathrm{V} 2$ & $124.78(18)$ & $\mathrm{C} 15-\mathrm{C} 16-\mathrm{H} 16 \mathrm{~A}$ & 110.1 \\
\hline $\mathrm{C} 25-\mathrm{N} 4-\mathrm{C} 31$ & $119.5(2)$ & $\mathrm{C} 15-\mathrm{C} 16-\mathrm{H} 16 \mathrm{~B}$ & 110.1 \\
\hline $\mathrm{C} 31-\mathrm{N} 4-\mathrm{V} 2$ & $115.70(15)$ & $\mathrm{H} 16 \mathrm{~A}-\mathrm{C} 16-\mathrm{H} 16 \mathrm{~B}$ & 108.4 \\
\hline $\mathrm{C} 14-\mathrm{N} 2-\mathrm{V} 1$ & $125.24(17)$ & $\mathrm{C} 4-\mathrm{C} 5-\mathrm{H} 5$ & 119.1 \\
\hline $\mathrm{C} 14-\mathrm{N} 2-\mathrm{C} 16$ & $118.9(2)$ & $\mathrm{C} 6-\mathrm{C} 5-\mathrm{C} 4$ & $121.8(2)$ \\
\hline $\mathrm{C} 16-\mathrm{N} 2-\mathrm{V} 1$ & $115.82(15)$ & $\mathrm{C} 6-\mathrm{C} 5-\mathrm{H} 5$ & 119.1 \\
\hline $\mathrm{C} 3-\mathrm{O} 1-\mathrm{V} 1$ & $131.62(15)$ & $\mathrm{C} 20-\mathrm{C} 21-\mathrm{H} 21$ & 119.3 \\
\hline $\mathrm{O} 2-\mathrm{C} 12-\mathrm{C} 9$ & $122.7(2)$ & $\mathrm{C} 19-\mathrm{C} 21-\mathrm{C} 20$ & $121.3(2)$ \\
\hline $\mathrm{O} 2-\mathrm{C} 12-\mathrm{C} 11$ & $118.8(2)$ & $\mathrm{C} 19-\mathrm{C} 21-\mathrm{H} 21$ & 119.3 \\
\hline $\mathrm{C} 11-\mathrm{C} 12-\mathrm{C} 9$ & $118.4(2)$ & $\mathrm{C} 12-\mathrm{C} 11-\mathrm{C} 10$ & $121.2(3)$ \\
\hline $\mathrm{C} 22-\mathrm{O} 4-\mathrm{V} 2$ & $131.37(15)$ & $\mathrm{C} 12-\mathrm{C} 11-\mathrm{H} 11$ & 119.4 \\
\hline $\mathrm{N} 2-\mathrm{C} 14-\mathrm{H} 14$ & 117.7 & $\mathrm{C} 10-\mathrm{C} 11-\mathrm{H} 11$ & 119.4 \\
\hline $\mathrm{N} 2-\mathrm{C} 14-\mathrm{C} 9$ & $124.5(2)$ & $\mathrm{C} 18-\mathrm{C} 19-\mathrm{H} 19$ & 120.7 \\
\hline C9-C14-H14 & 117.7 & $\mathrm{C} 21-\mathrm{C} 19-\mathrm{C} 18$ & $118.7(2)$ \\
\hline $\mathrm{O} 6-\mathrm{C} 30-\mathrm{C} 24$ & $123.4(2)$ & $\mathrm{C} 21-\mathrm{C} 19-\mathrm{H} 19$ & 120.7 \\
\hline $\mathrm{O} 6-\mathrm{C} 30-\mathrm{C} 29$ & $119.0(2)$ & $\mathrm{C} 26-\mathrm{C} 27-\mathrm{C} 28$ & $119.0(3)$ \\
\hline $\mathrm{C} 29-\mathrm{C} 30-\mathrm{C} 24$ & $117.6(2)$ & $\mathrm{C} 26-\mathrm{C} 27-\mathrm{H} 27$ & 120.5 \\
\hline $\mathrm{O} 1-\mathrm{C} 3-\mathrm{C} 4$ & $123.5(2)$ & $\mathrm{C} 28-\mathrm{C} 27-\mathrm{H} 27$ & 120.5 \\
\hline $\mathrm{O} 1-\mathrm{C} 3-\mathrm{C} 2$ & $118.9(2)$ & $\mathrm{C} 1-\mathrm{C} 6-\mathrm{H} 6$ & 120.8 \\
\hline $\mathrm{C} 2-\mathrm{C} 3-\mathrm{C} 4$ & $117.6(2)$ & $\mathrm{C} 5-\mathrm{C} 6-\mathrm{C} 1$ & $118.4(2)$ \\
\hline $\mathrm{N} 4-\mathrm{C} 25-\mathrm{H} 25$ & 117.3 & $\mathrm{C} 5-\mathrm{C} 6-\mathrm{H} 6$ & 120.8 \\
\hline $\mathrm{N} 4-\mathrm{C} 25-\mathrm{C} 24$ & $125.4(2)$ & $\mathrm{C} 30-\mathrm{C} 29-\mathrm{H} 29$ & 119.3 \\
\hline $\mathrm{C} 24-\mathrm{C} 25-\mathrm{H} 25$ & 117.3 & $\mathrm{C} 28-\mathrm{C} 29-\mathrm{C} 30$ & $121.4(3)$ \\
\hline $\mathrm{C} 15-\mathrm{N} 1-\mathrm{V} 1$ & $111.60(15)$ & $\mathrm{C} 28-\mathrm{C} 29-\mathrm{H} 29$ & 119.3 \\
\hline $\mathrm{C} 13-\mathrm{N} 1-\mathrm{V} 1$ & $127.96(17)$ & $\mathrm{N} 4-\mathrm{C} 31-\mathrm{H} 31 \mathrm{~A}$ & 110.0 \\
\hline $\mathrm{C} 13-\mathrm{N} 1-\mathrm{C} 15$ & $120.3(2)$ & $\mathrm{N} 4-\mathrm{C} 31-\mathrm{H} 31 \mathrm{~B}$ & 110.0 \\
\hline $\mathrm{C} 24-\mathrm{C} 26-\mathrm{H} 26$ & 119.2 & $\mathrm{~N} 4-\mathrm{C} 31-\mathrm{C} 32$ & $108.60(19)$ \\
\hline $\mathrm{C} 27-\mathrm{C} 26-\mathrm{H} 26$ & 119.2 & $\mathrm{H} 31 \mathrm{~A}-\mathrm{C} 31-\mathrm{H} 31 \mathrm{~B}$ & 108.4 \\
\hline $\mathrm{C} 27-\mathrm{C} 26-\mathrm{C} 24$ & $121.5(3)$ & $\mathrm{C} 32-\mathrm{C} 31-\mathrm{H} 31 \mathrm{~A}$ & 110.0 \\
\hline $\mathrm{C} 23-\mathrm{N} 3-\mathrm{V} 2$ & $127.69(17)$ & $\mathrm{C} 32-\mathrm{C} 31-\mathrm{H} 31 \mathrm{~B}$ & 110.0 \\
\hline $\mathrm{C} 23-\mathrm{N} 3-\mathrm{C} 32$ & $120.6(2)$ & $\mathrm{N} 3-\mathrm{C} 32-\mathrm{C} 31$ & $106.66(19)$ \\
\hline $\mathrm{C} 32-\mathrm{N} 3-\mathrm{V} 2$ & $111.60(15)$ & $\mathrm{N} 3-\mathrm{C} 32-\mathrm{H} 32 \mathrm{~A}$ & 110.4 \\
\hline $\mathrm{C} 3-\mathrm{C} 4-\mathrm{C} 13$ & $122.6(2)$ & $\mathrm{N} 3-\mathrm{C} 32-\mathrm{H} 32 \mathrm{~B}$ & 110.4 \\
\hline $\mathrm{C} 5-\mathrm{C} 4-\mathrm{C} 3$ & $119.7(2)$ & $\mathrm{C} 31-\mathrm{C} 32-\mathrm{H} 32 \mathrm{~A}$ & 110.4 \\
\hline $\mathrm{C} 5-\mathrm{C} 4-\mathrm{C} 13$ & $117.6(2)$ & $\mathrm{C} 31-\mathrm{C} 32-\mathrm{H} 32 \mathrm{~B}$ & 110.4 \\
\hline $\mathrm{C} 7-\mathrm{C} 10-\mathrm{H} 10$ & 119.7 & $\mathrm{H} 32 \mathrm{~A}-\mathrm{C} 32-\mathrm{H} 32 \mathrm{~B}$ & 108.6 \\
\hline $\mathrm{C} 7-\mathrm{C} 10-\mathrm{C} 11$ & $120.5(3)$ & $\mathrm{C} 34-\mathrm{O} 8-\mathrm{H} 8 \mathrm{~A}$ & 109.5 \\
\hline $\mathrm{C} 11-\mathrm{C} 10-\mathrm{H} 10$ & 119.7 & $\mathrm{C} 33-\mathrm{O} 7-\mathrm{H} 7 \mathrm{~A}$ & 109.5 \\
\hline $\mathrm{C} 9-\mathrm{C} 8-\mathrm{H} 8$ & 119.3 & $\mathrm{O} 7-\mathrm{C} 33-\mathrm{H} 33 \mathrm{~A}$ & 109.5 \\
\hline $\mathrm{C} 7-\mathrm{C} 8-\mathrm{H} 8$ & 119.3 & $\mathrm{O} 7-\mathrm{C} 33-\mathrm{H} 33 \mathrm{~B}$ & 109.5 \\
\hline $\mathrm{C} 7-\mathrm{C} 8-\mathrm{C} 9$ & $121.4(3)$ & $\mathrm{O} 7-\mathrm{C} 33-\mathrm{H} 33 \mathrm{C}$ & 109.5 \\
\hline $\mathrm{N} 1-\mathrm{C} 15-\mathrm{H} 15 \mathrm{~A}$ & 110.5 & $\mathrm{H} 33 \mathrm{~A}-\mathrm{C} 33-\mathrm{H} 33 \mathrm{~B}$ & 109.5 \\
\hline $\mathrm{N} 1-\mathrm{C} 15-\mathrm{H} 15 \mathrm{~B}$ & 110.5 & $\mathrm{H} 33 \mathrm{~A}-\mathrm{C} 33-\mathrm{H} 33 \mathrm{C}$ & 109.5 \\
\hline
\end{tabular}




\begin{tabular}{|c|c|c|c|}
\hline $\mathrm{N} 1-\mathrm{C} 15-\mathrm{C} 16$ & $106.33(19)$ & $\mathrm{H} 33 \mathrm{~B}-\mathrm{C} 33-\mathrm{H} 33 \mathrm{C}$ & 109.5 \\
\hline $\mathrm{H} 15 \mathrm{~A}-\mathrm{C} 15-\mathrm{H} 15 \mathrm{~B}$ & 108.7 & $\mathrm{O} 8-\mathrm{C} 34-\mathrm{H} 34 \mathrm{~A}$ & 109.5 \\
\hline $\mathrm{C} 16-\mathrm{C} 15-\mathrm{H} 15 \mathrm{~A}$ & 110.5 & $\mathrm{O} 8-\mathrm{C} 34-\mathrm{H} 34 \mathrm{~B}$ & 109.5 \\
\hline $\mathrm{C} 16-\mathrm{C} 15-\mathrm{H} 15 \mathrm{~B}$ & 110.5 & $\mathrm{O} 8-\mathrm{C} 34-\mathrm{H} 34 \mathrm{C}$ & 109.5 \\
\hline $\mathrm{O} 4-\mathrm{C} 22-\mathrm{C} 17$ & $118.7(2)$ & $\mathrm{H} 34 \mathrm{~A}-\mathrm{C} 34-\mathrm{H} 34 \mathrm{~B}$ & 109.5 \\
\hline $\mathrm{O} 4-\mathrm{C} 22-\mathrm{C} 20$ & $123.8(2)$ & $\mathrm{H} 34 \mathrm{~A}-\mathrm{C} 34-\mathrm{H} 34 \mathrm{C}$ & 109.5 \\
\hline $\mathrm{C} 17-\mathrm{C} 22-\mathrm{C} 20$ & $117.5(2)$ & $\mathrm{H} 34 \mathrm{~B}-\mathrm{C} 34-\mathrm{H} 34 \mathrm{C}$ & 109.5 \\
\hline $\mathrm{C} 22-\mathrm{C} 17-\mathrm{H} 17$ & 119.2 & $\mathrm{C} 33 \mathrm{~A}-\mathrm{O} 7 \mathrm{~A}-\mathrm{H} 7 \mathrm{AA}$ & 109.5 \\
\hline $\mathrm{C} 18-\mathrm{C} 17-\mathrm{C} 22$ & $121.5(2)$ & $\mathrm{O} 7 \mathrm{~A}-\mathrm{C} 33 \mathrm{~A}-\mathrm{H} 33 \mathrm{D}$ & 109.5 \\
\hline $\mathrm{C} 18-\mathrm{C} 17-\mathrm{H} 17$ & 119.2 & $\mathrm{O} 7 \mathrm{~A}-\mathrm{C} 33 \mathrm{~A}-\mathrm{H} 33 \mathrm{E}$ & 109.5 \\
\hline $\mathrm{C} 30-\mathrm{C} 24-\mathrm{C} 25$ & $121.7(2)$ & $\mathrm{O} 7 \mathrm{~A}-\mathrm{C} 33 \mathrm{~A}-\mathrm{H} 33 \mathrm{~F}$ & 109.5 \\
\hline $\mathrm{C} 26-\mathrm{C} 24-\mathrm{C} 30$ & $119.7(2)$ & $\mathrm{H} 33 \mathrm{D}-\mathrm{C} 33 \mathrm{~A}-\mathrm{H} 33 \mathrm{E}$ & 109.5 \\
\hline $\mathrm{C} 26-\mathrm{C} 24-\mathrm{C} 25$ & $118.6(2)$ & $\mathrm{H} 33 \mathrm{D}-\mathrm{C} 33 \mathrm{~A}-\mathrm{H} 33 \mathrm{~F}$ & 109.5 \\
\hline $\mathrm{N} 1-\mathrm{C} 13-\mathrm{C} 4$ & $124.1(2)$ & $\mathrm{H} 33 \mathrm{E}-\mathrm{C} 33 \mathrm{~A}-\mathrm{H} 33 \mathrm{~F}$ & 109.5 \\
\hline $\mathrm{N} 1-\mathrm{C} 13-\mathrm{H} 13$ & 117.9 & $\mathrm{H} 34 \mathrm{D}-\mathrm{C} 34 \mathrm{~A}-\mathrm{H} 34 \mathrm{E}$ & 109.5 \\
\hline $\mathrm{C} 4-\mathrm{C} 13-\mathrm{H} 13$ & 117.9 & $\mathrm{H} 34 \mathrm{D}-\mathrm{C} 34 \mathrm{~A}-\mathrm{H} 34 \mathrm{~F}$ & 109.5 \\
\hline $\mathrm{C} 17-\mathrm{C} 18-\mathrm{H} 18$ & 119.4 & $\mathrm{H} 34 \mathrm{E}-\mathrm{C} 34 \mathrm{~A}-\mathrm{H} 34 \mathrm{~F}$ & 109.5 \\
\hline $\mathrm{C} 17-\mathrm{C} 18-\mathrm{C} 19$ & $121.2(3)$ & $\mathrm{O} 8 \mathrm{~A}-\mathrm{C} 34 \mathrm{~A}-\mathrm{H} 34 \mathrm{D}$ & 109.5 \\
\hline $\mathrm{C} 19-\mathrm{C} 18-\mathrm{H} 18$ & 119.4 & $\mathrm{O} 8 \mathrm{~A}-\mathrm{C} 34 \mathrm{~A}-\mathrm{H} 34 \mathrm{E}$ & 109.5 \\
\hline $\mathrm{C} 2-\mathrm{C} 1-\mathrm{H} 1$ & 119.4 & $\mathrm{O} 8 \mathrm{~A}-\mathrm{C} 34 \mathrm{~A}-\mathrm{H} 34 \mathrm{~F}$ & 109.5 \\
\hline $\mathrm{C} 2-\mathrm{C} 1-\mathrm{C} 6$ & $121.2(3)$ & $\mathrm{C} 34 \mathrm{~A}-\mathrm{O} 8 \mathrm{~A}-\mathrm{H} 8 \mathrm{AA}$ & 109.5 \\
\hline $\mathrm{V} 2-\mathrm{O} 6-\mathrm{C} 30-\mathrm{C} 24$ & $-29.7(3)$ & $\mathrm{C} 4-\mathrm{C} 5-\mathrm{C} 6-\mathrm{C} 1$ & $-2.1(4)$ \\
\hline $\mathrm{V} 2-\mathrm{O} 6-\mathrm{C} 30-\mathrm{C} 29$ & $151.31(17)$ & $\mathrm{C} 15-\mathrm{N} 1-\mathrm{C} 13-\mathrm{C} 4$ & $177.3(2)$ \\
\hline $\mathrm{V} 2-\mathrm{N} 4-\mathrm{C} 25-\mathrm{C} 24$ & $7.9(3)$ & $\mathrm{C} 22-\mathrm{C} 17-\mathrm{C} 18-\mathrm{C} 19$ & $-0.6(4)$ \\
\hline $\mathrm{V} 2-\mathrm{N} 4-\mathrm{C} 31-\mathrm{C} 32$ & $22.2(2)$ & $\mathrm{C} 22-\mathrm{C} 20-\mathrm{C} 23-\mathrm{N} 3$ & $-7.4(4)$ \\
\hline $\mathrm{V} 2-\mathrm{O} 4-\mathrm{C} 22-\mathrm{C} 17$ & $-170.69(17)$ & $\mathrm{C} 22-\mathrm{C} 20-\mathrm{C} 21-\mathrm{C} 19$ & $-1.0(3)$ \\
\hline $\mathrm{V} 2-\mathrm{O} 4-\mathrm{C} 22-\mathrm{C} 20$ & $11.4(3)$ & $\mathrm{C} 17-\mathrm{C} 22-\mathrm{C} 20-\mathrm{C} 23$ & $-172.0(2)$ \\
\hline $\mathrm{V} 2-\mathrm{N} 3-\mathrm{C} 23-\mathrm{C} 20$ & $-7.0(3)$ & $\mathrm{C} 17-\mathrm{C} 22-\mathrm{C} 20-\mathrm{C} 21$ & $3.7(3)$ \\
\hline $\mathrm{V} 2-\mathrm{N} 3-\mathrm{C} 32-\mathrm{C} 31$ & $46.0(2)$ & $\mathrm{C} 17-\mathrm{C} 18-\mathrm{C} 19-\mathrm{C} 21$ & $3.4(4)$ \\
\hline $\mathrm{V} 1-\mathrm{O} 2-\mathrm{C} 12-\mathrm{C} 9$ & $-29.3(3)$ & $\mathrm{C} 24-\mathrm{C} 30-\mathrm{C} 29-\mathrm{C} 28$ & $1.1(3)$ \\
\hline $\mathrm{V} 1-\mathrm{O} 2-\mathrm{C} 12-\mathrm{C} 11$ & $153.17(16)$ & $\mathrm{C} 24-\mathrm{C} 26-\mathrm{C} 27-\mathrm{C} 28$ & $1.1(4)$ \\
\hline $\mathrm{V} 1-\mathrm{N} 2-\mathrm{C} 14-\mathrm{C} 9$ & $7.7(3)$ & $\mathrm{C} 13-\mathrm{N} 1-\mathrm{C} 15-\mathrm{C} 16$ & $-137.4(2)$ \\
\hline $\mathrm{V} 1-\mathrm{N} 2-\mathrm{C} 16-\mathrm{C} 15$ & $22.8(2)$ & $\mathrm{C} 13-\mathrm{C} 4-\mathrm{C} 5-\mathrm{C} 6$ & $175.5(2)$ \\
\hline $\mathrm{V} 1-\mathrm{O} 1-\mathrm{C} 3-\mathrm{C} 4$ & $10.4(3)$ & $\mathrm{C} 9-\mathrm{C} 12-\mathrm{C} 11-\mathrm{C} 10$ & $1.9(3)$ \\
\hline $\mathrm{V} 1-\mathrm{O} 1-\mathrm{C} 3-\mathrm{C} 2$ & $-170.93(17)$ & $\mathrm{C} 9-\mathrm{C} 8-\mathrm{C} 7-\mathrm{C} 10$ & $1.7(4)$ \\
\hline $\mathrm{V} 1-\mathrm{N} 1-\mathrm{C} 15-\mathrm{C} 16$ & $46.5(2)$ & $\mathrm{C} 7-\mathrm{C} 10-\mathrm{C} 11-\mathrm{C} 12$ & $-0.5(4)$ \\
\hline $\mathrm{V} 1-\mathrm{N} 1-\mathrm{C} 13-\mathrm{C} 4$ & $-7.2(3)$ & $\mathrm{C} 7-\mathrm{C} 8-\mathrm{C} 9-\mathrm{C} 12$ & $-0.2(4)$ \\
\hline $\mathrm{O} 6-\mathrm{C} 30-\mathrm{C} 24-\mathrm{C} 25$ & $-1.8(3)$ & $\mathrm{C} 7-\mathrm{C} 8-\mathrm{C} 9-\mathrm{C} 14$ & $-176.6(2)$ \\
\hline $\mathrm{O} 6-\mathrm{C} 30-\mathrm{C} 24-\mathrm{C} 26$ & $-179.2(2)$ & $\mathrm{C} 20-\mathrm{C} 22-\mathrm{C} 17-\mathrm{C} 18$ & $-3.0(4)$ \\
\hline $\mathrm{O} 6-\mathrm{C} 30-\mathrm{C} 29-\mathrm{C} 28$ & $-179.9(2)$ & $\mathrm{C} 20-\mathrm{C} 21-\mathrm{C} 19-\mathrm{C} 18$ & $-2.6(4)$ \\
\hline $\mathrm{O} 2-\mathrm{C} 12-\mathrm{C} 9-\mathrm{C} 14$ & $-2.8(3)$ & $\mathrm{C} 2-\mathrm{C} 3-\mathrm{C} 4-\mathrm{C} 13$ & $-172.5(2)$ \\
\hline $\mathrm{O} 2-\mathrm{C} 12-\mathrm{C} 9-\mathrm{C} 8$ & $-179.1(2)$ & $\mathrm{C} 2-\mathrm{C} 3-\mathrm{C} 4-\mathrm{C} 5$ & $3.4(3)$ \\
\hline $\mathrm{O} 2-\mathrm{C} 12-\mathrm{C} 11-\mathrm{C} 10$ & $179.6(2)$ & $\mathrm{C} 2-\mathrm{C} 1-\mathrm{C} 6-\mathrm{C} 5$ & $1.9(4)$ \\
\hline $\mathrm{N} 4-\mathrm{C} 25-\mathrm{C} 24-\mathrm{C} 30$ & $12.3(4)$ & $\mathrm{C} 23-\mathrm{N} 3-\mathrm{C} 32-\mathrm{C} 31$ & $-137.4(2)$ \\
\hline $\mathrm{N} 4-\mathrm{C} 25-\mathrm{C} 24-\mathrm{C} 26$ & $-170.2(2)$ & $\mathrm{C} 23-\mathrm{C} 20-\mathrm{C} 21-\mathrm{C} 19$ & $174.9(2)$ \\
\hline $\mathrm{N} 4-\mathrm{C} 31-\mathrm{C} 32-\mathrm{N} 3$ & $-42.8(2)$ & $\mathrm{C} 16-\mathrm{N} 2-\mathrm{C} 14-\mathrm{C} 9$ & $-174.5(2)$ \\
\hline $\mathrm{N} 2-\mathrm{C} 14-\mathrm{C} 9-\mathrm{C} 12$ & $13.4(3)$ & $\mathrm{C} 5-\mathrm{C} 4-\mathrm{C} 13-\mathrm{N} 1$ & $176.9(2)$ \\
\hline
\end{tabular}




$\begin{array}{ll}\mathrm{N} 2-\mathrm{C} 14-\mathrm{C} 9-\mathrm{C} 8 & -170.3(2) \\ \mathrm{O} 1-\mathrm{C} 3-\mathrm{C} 4-\mathrm{C} 13 & 6.2(3) \\ \mathrm{O} 1-\mathrm{C} 3-\mathrm{C} 4-\mathrm{C} 5 & -177.9(2) \\ \mathrm{O} 1-\mathrm{C} 3-\mathrm{C} 2-\mathrm{C} 1 & 177.6(2) \\ \mathrm{O} 4-\mathrm{C} 22-\mathrm{C} 17-\mathrm{C} 18 & 179.0(2) \\ \mathrm{O} 4-\mathrm{C} 22-\mathrm{C} 20-\mathrm{C} 23 & 5.9(3) \\ \mathrm{O} 4-\mathrm{C} 22-\mathrm{C} 20-\mathrm{C} 21 & -178.4(2) \\ \mathrm{C} 14-\mathrm{N} 2-\mathrm{C} 16-\mathrm{C} 15 & -155.1(2) \\ \mathrm{C} 3-\mathrm{C} 4-\mathrm{C} 13-\mathrm{N} 1 & -7.1(4) \\ \mathrm{C} 3-\mathrm{C} 4-\mathrm{C} 5-\mathrm{C} 6 & -0.6(3) \\ \mathrm{C} 25-\mathrm{N} 4-\mathrm{C} 31-\mathrm{C} 32 & -155.7(2) \\ \mathrm{N} 1-\mathrm{C} 15-\mathrm{C} 16-\mathrm{N} 2 & -43.4(2) \\ \mathrm{C} 4-\mathrm{C} 3-\mathrm{C} 2-\mathrm{C} 1 & -3.7(4)\end{array}$

$\mathrm{C} 21-\mathrm{C} 20-\mathrm{C} 23-\mathrm{N} 3$
$\mathrm{C} 11-\mathrm{C} 12-\mathrm{C} 9-\mathrm{C} 14$
$\mathrm{C} 11-\mathrm{C} 12-\mathrm{C} 9-\mathrm{C} 8$
$\mathrm{C} 11-\mathrm{C} 10-\mathrm{C} 7-\mathrm{C} 8$
$\mathrm{C} 27-\mathrm{C} 26-\mathrm{C} 24-\mathrm{C} 30$
$\mathrm{C} 27-\mathrm{C} 26-\mathrm{C} 24-\mathrm{C} 25$
$\mathrm{C} 27-\mathrm{C} 28-\mathrm{C} 29-\mathrm{C} 30$
$\mathrm{C} 6-\mathrm{C} 1-\mathrm{C} 2-\mathrm{C} 3$
$\mathrm{C} 29-\mathrm{C} 30-\mathrm{C} 24-\mathrm{C} 25$
$\mathrm{C} 29-\mathrm{C} 30-\mathrm{C} 24-\mathrm{C} 26$
$\mathrm{C} 29-\mathrm{C} 28-\mathrm{C} 27-\mathrm{C} 26$
$\mathrm{C} 31-\mathrm{N} 4-\mathrm{C} 25-\mathrm{C} 24$
$\mathrm{C} 32-\mathrm{N} 3-\mathrm{C} 23-\mathrm{C} 20$

$176.9(2)$

$174.7(2)$

$-1.6(3)$

$-1.3(4)$

$-0.8(4)$

$-178.4(2)$

$-0.8(4)$

$1.1(4)$

$177.2(2)$

$-0.3(3)$

$-0.3(4)$

$-174.5(2)$

$177.0(2)$

Hydrogen-bond geometry $\left(\AA,{ }^{\circ}\right)$

\begin{tabular}{lllll}
\hline$D-\mathrm{H} \cdots A$ & $D-\mathrm{H}$ & $\mathrm{H} \cdots A$ & $D \cdots A$ & $D-\mathrm{H} \cdots A$ \\
\hline $\mathrm{C} 14-\mathrm{H} 14 \cdots \mathrm{O} 8^{\mathrm{i}}$ & 0.93 & 2.48 & $3.368(4)$ & 159 \\
$\mathrm{C} 14-\mathrm{H} 14 \cdots \mathrm{O} 8 A^{\mathrm{i}}$ & 0.93 & 2.48 & $3.299(7)$ & 147 \\
$\mathrm{C} 25-\mathrm{H} 25 \cdots \mathrm{O} 7^{\mathrm{ii}}$ & 0.93 & 2.51 & $3.391(4)$ & 158 \\
$\mathrm{C} 25-\mathrm{H} 25 \cdots \mathrm{O} 7 A^{\mathrm{ii}}$ & 0.93 & 2.66 & $3.457(9)$ & 144 \\
$\mathrm{C} 13-\mathrm{H} 13 \cdots \mathrm{O} 3^{\mathrm{iii}}$ & 0.93 & 2.60 & $3.372(3)$ & 141 \\
$\mathrm{C} 23-\mathrm{H} 23 \cdots \mathrm{O} 5^{\text {iv }}$ & 0.93 & 2.59 & $3.364(3)$ & 141 \\
$\mathrm{O} 8-\mathrm{H} 8 A \cdots \mathrm{O} 2$ & 0.82 & 2.12 & $2.926(4)$ & 167 \\
$\mathrm{O} 7-\mathrm{H} 7 A \cdots \mathrm{O} 6$ & 0.82 & 2.18 & $2.940(3)$ & 153 \\
$\mathrm{O} 7-\mathrm{H} 7 A \cdots \mathrm{O} 4$ & 0.82 & 2.64 & $3.246(4)$ & 132 \\
$\mathrm{O} 8 A-\mathrm{H} 8 A A \cdots \mathrm{O} 1$ & 0.82 & 2.18 & $2.984(6)$ & 166 \\
\hline
\end{tabular}

Symmetry codes: (i) $x, y+1, z$; (ii) $x, y-1, z$; (iii) $x-1 / 2,-y+5 / 2, z$; (iv) $x-1 / 2,-y+1 / 2, z$. 